Article

\title{
The Synthesis of Some Perhydrobenzimidazolinium Salts and Their Application in Pd-Carbene Catalyzed Heck and Suzuki Reactions
}

\author{
Murat Yiğit \\ Department of Chemistry, Faculty of Science and Art, Adıyaman University, 02040 Adryaman, \\ Turkey; E-Mail: myigit@adiyaman.edu.tr; Fax: +90 4162231774
}

Received: 13 May 2009; in revised form: 27 May 2009 / Accepted: 3 June 2009 /

Published: 5 June 2009

\begin{abstract}
Novel 1,3-dialkylperhydrobenzimidazolinium chloride salts were prepared as precursors of $N$-heterocyclic carbenes 3a-e by reacting $N, N^{\prime}$-dialkylcyclohexandiamine, triethyl orthoformate and ammonium chloride. The salts were characterized spectroscopically and the complexes formed in situ from $\mathrm{Pd}(\mathrm{OAc})_{2}$ and $\mathbf{3}$ have been tested as catalysts in homogenous Heck and Suzuki reactions.
\end{abstract}

Keyword: Heck and Suzuki reactions; perhydrobenzimidazolinium salt; palladium; carbene; catalyst

\section{Introduction}

Palladium-catalyzed C-C bond formation is one of the most fundemental and important reactions in modern synthetic chemistry [1,2]. It represents the key the step in a wide range of preparative organic processes, from the synthesis of natural products [3] to supramolecular chemistry and material science [4]. Common methodologies used are the palladium mediated coupling of the organic halides or halide equvalents with Grignard reagents, organotin or organoboron reagents where monodentate phosphine are usually employed as ancillary ligands [5-8]. Recently, $N$-heterocyclic carbenes (NHCs) have been used as alternative or better ancillary ligands than phosphine. The major advantage of NHCs in catalysis is that an increased stability of the active catalyst is often observed, allowing for lower operational catalyst loadings, prolonged reaction times and tolerance of increased reaction temperatures [9-11]. The Heck and Suzuki couplings are fascinating reactions from a catalysis science perspective. Virtually all forms of palladium can be used as precatalysts for the simpler reactions, yet 
specifically designed catalysts are required for activation of bulky or electronically unactivated substrates. Additionally, in many cases, extremely small amounts of palladium (ppm or ppb levels) are sufficient to give very high turnover frequencies, whereas in others, $10 \%$ or higher precatalyst loadings are required to obtain adequate product yields [12]. Initially, water-soluble phosphines were used as ligands for the cross-coupling reactions in aqueous media [13], but in recent years, other hydrophilic phosphine-free systems [14] and soluble palladium nanoparticles [15-17] have also been found to be higly efficient catalysts for this transformation. The use of water as a solvent for chemical reactions clearly has both economical and environmental advantages because it is inexpensive, abundant, nontoxic, nonflammable and readily separable from organic compounds [18]. There have been a number of reports of the palladium-mediated Heck and Suzuki reaction being performed using water as solvent [19-27]. Due to the large number of Suzuki and other coupling reactions that are carried out in aqueous [19] or biphasic systems, there has been an increased interest in the development of watersoluble ligands for these reactions. Shaughnessy and co-workers utilized both sterically demanding water-soluble alkylphosphines [28] and triarylphosphines [29] in Suzuki and Heck couplings of aryl bromides, respectively. We have previously reported the use of the various catalytic systems, such as palladium catalyzed cross-coupling, ruthenium catalyzed hydrogenation and rhodium catalyzed arylation or hydrosilylation [30-34]. Although the nature of the NHC ligand on complexes has a tremendous influence on the rate of catalyzed reactions, the use of perhydrobenzimidazolinium ligands in Heck and Suzuki reactions is a neglected field. In order to find more efficient catalyst, we have prepared a series of new perhydrobenzimidazolinium chlorides, 3a-e (Scheme 1), containing a saturated benzimidazole ring and we report here an in situ palladium-carbene based catalytic system for Heck and Suzuki cross-coupling of aryl halides.

\section{Results and Discussion}

The general route to the $p$-substituent containing ligand precursors is shown in Scheme 1. The condensation of 1,2-diaminocyclohexane with aldehydes in ethanol gave the corresponding Schiff bases in high yields. Reduction of these Schiff bases with $\mathrm{NaBH}_{4}$ in methanol leads to $N$, $N$ '-dialkylcyclohexan-1,2-diamines. The symmetrical 1,3-dialkylperhydrobenzimidazolinium salts 3 were easly synthesized in high yields from the $N, N^{\prime}$-dialkylcyclohexan-1,2-diamines, triethyl ortoformate and ammonium chloride. After purification, the 1,3-dialkylperhydrobenzimidazolinium salts 3a-e were obtained in good yields (85-90\%). The salts are soluble in the common polar solvents ethanol and dichloromethane and are stable under air and in the presence of moisture. The structures of 3 were determined by their spectroscopic data and elemental analyses (see Experimental section). The ${ }^{13} \mathrm{C}$ NMR spectra of 3a-e show only sharp high field signals [159.5 ppm (3a), 162.1 ppm (3b), 161.9 ppm (3c), $162.5 \mathrm{ppm}$ (3d), $162.0 \mathrm{ppm}$ (3e)] for the imino carbon and for a benzylic carbon [58.7 ppm (3a),

$66.7 \mathrm{ppm}(\mathbf{3 b}), 66.6 \mathrm{ppm}(\mathbf{3 c}), 67.0 \mathrm{ppm}(\mathbf{3 d}), 66.8 \mathrm{ppm}(\mathbf{3 e})]$. The ${ }^{1} \mathrm{H}-\mathrm{NMR}$ spectra of the perhydrobenzimidazolinium salts further supported the assigned structures. The resonances of the $\mathrm{C}(2)-\mathrm{H}$ were observed as sharp singlets at $\delta=10.87,10.96,10.74,11.10,10.79 \mathrm{ppm}$ for 3a-e, respectively. The benzylic protons appeared as a doublet at $4.36-5.50 \mathrm{ppm}$. The IR data for perhydrobenzimidazolinium 3a-e salts clearly indicate the presence of the $-\mathrm{C}=\mathrm{N}$ - group with a $v_{(\mathrm{C}=\mathrm{N})}$ vibration at 1,644, 1,592, 1,612, 1,587 and 1,600 $\mathrm{cm}^{-1}$ for 3a-e, respectively. These NMR and IR values were similar to those reproted for other 1,3-dialkylperhydrobenzimidazolinium and 1,3-dialkylimidazolinium salts [33,35]. 
Scheme 1. Synthesis of 1,3-dialkylperhydrobenzimidazolinium salts.

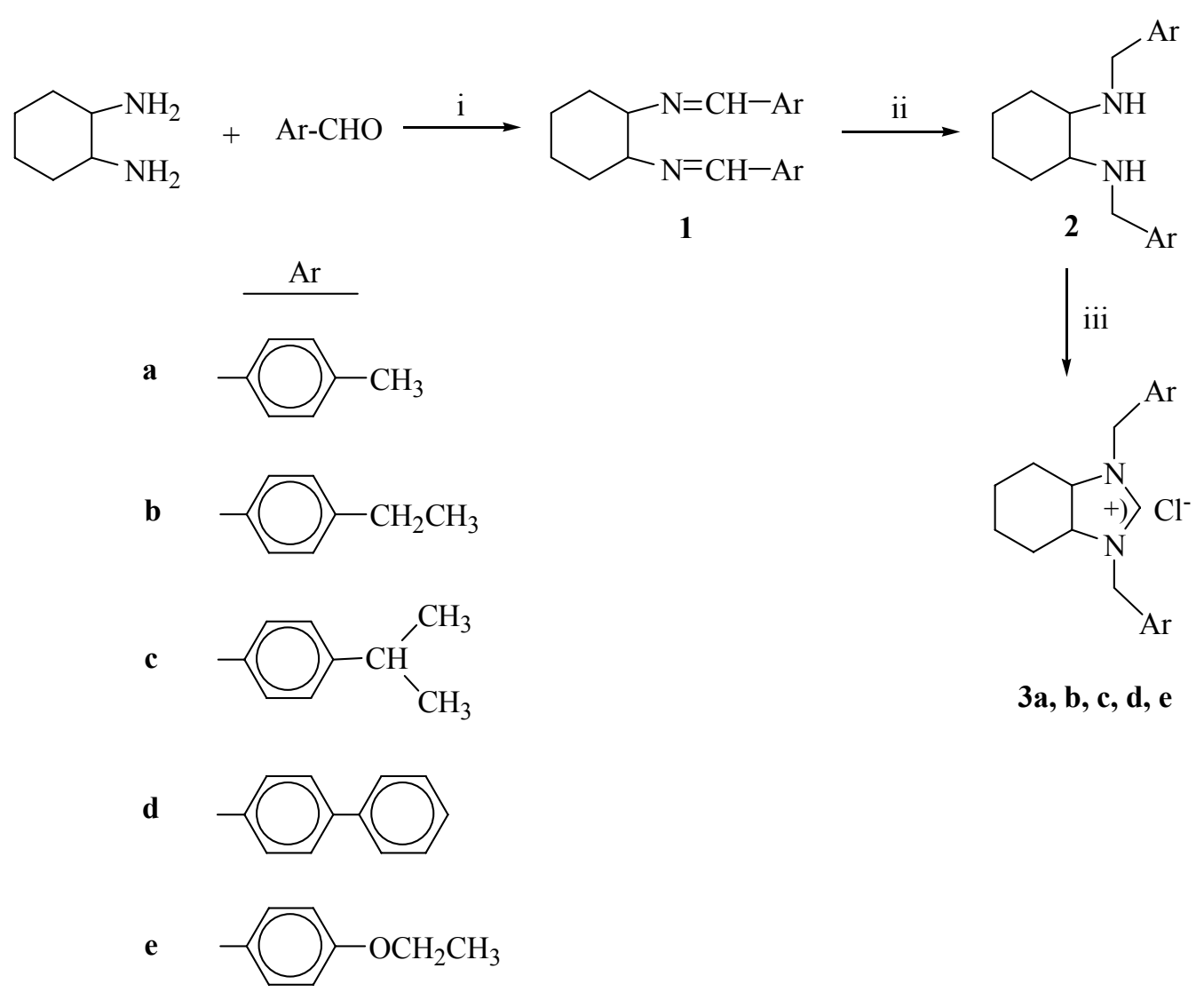

Reagents and conditions: (i) EtOH, 3h, reflux; (ii) $\mathrm{MeOH}_{2} \mathrm{NaBH}_{4}, 5 \mathrm{~h}$, RT; (iii) $\mathrm{HC}(\mathrm{OEt})_{3}, \mathrm{NH}_{4} \mathrm{CI}$, $12 \mathrm{~h}, 110^{\circ} \mathrm{C}$.

The Pd-catalyzed Heck reaction is an efficient way to prepare styrene derivatives, which are important chemicals for many applications. The Heck couplings of styrene with aryl bromides to form alkenes were undertaken with $\mathrm{Pd}(\mathrm{OAc})_{2} / \mathbf{3 a - e}$ as catalysts. The choice of bases and solvents are usually important in achieving an efficient cross coupling reaction. The widely used solvents such as DMF, DMSO, toluene, dioxane, THF and $\mathrm{CH}_{3} \mathrm{CN}$ had remarkable effects on the coupling reaction. For optimal reaction conditions, the $\mathrm{Pd}(\mathrm{OAc})_{2}$-catalyzed cross coupling of bromobenzene with styrene was employed as the model reaction using ligand 3a at $80{ }^{\circ} \mathrm{C} ; \mathrm{Cs}_{2} \mathrm{CO}_{3}, \mathrm{~K}_{2} \mathrm{CO}_{3}, \mathrm{~K}_{3} \mathrm{PO}_{4}$ and $t-\mathrm{BuOK}$ were tested as the base. The coupling reactions of aryl bromides and styrene were carried out in $\mathrm{DMF} / \mathrm{H}_{2} \mathrm{O}$ (3:3 mL) with $1 \mathrm{~mol} \% \mathrm{Pd}(\mathrm{OAc})_{2}, 2 \mathrm{~mol} \% 3$ and 2 equiv. $\mathrm{K}_{2} \mathrm{CO}_{3}$ for $2 \mathrm{~h}$ at $80^{\circ} \mathrm{C}$. The results are summarized in Table 1.

Control experiments indicate that the coupling reactions did not occur in the absence of 3a. Under these reaction conditions, a wide range of aryl bromides bearing electron-donating or electronwithdrawing groups react with styrene affording the coupled products in excellent yields (Table 1, entries 3, 4, 8, 15, 18 and 22). Enhancements in activity, although less significant, are also observed employing 4-bromobenzaldehyde instead of 4-bromoacetophenone (entries 1-5 and 11-15, respectively). 
Table 1. The Heck coupling reaction of aryl bromides with styrene.

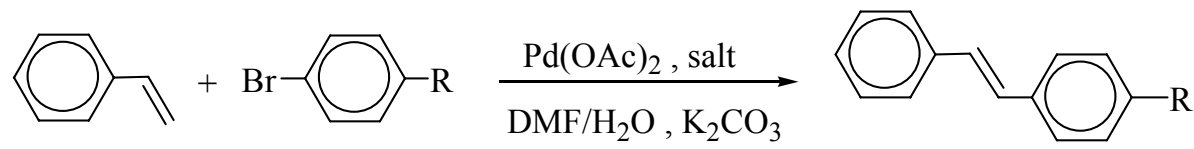

\begin{tabular}{|c|c|c|c|}
\hline Entry & $\mathbf{R}$ & SALT & Yield $(\%)^{b, c}$ \\
\hline 1 & $\mathrm{COCH}_{3}$ & $\mathbf{3 a}$ & 96 \\
\hline 2 & $\mathrm{COCH}_{3}$ & $3 b$ & 95 \\
\hline 3 & $\mathrm{COCH}_{3}$ & $3 c$ & 98 \\
\hline 4 & $\mathrm{COCH}_{3}$ & 3d & 98 \\
\hline 5 & $\mathrm{COCH}_{3}$ & $\mathbf{3 e}$ & 96 \\
\hline 6 & $\mathrm{CHO}$ & $\mathbf{3 a}$ & 95 \\
\hline 7 & $\mathrm{CHO}$ & $\mathbf{3 b}$ & 94 \\
\hline 8 & $\mathrm{CHO}$ & $3 c$ & 96 \\
\hline 9 & $\mathrm{CHO}$ & 3d & 91 \\
\hline 10 & $\mathrm{CHO}$ & $3 \mathbf{e}$ & 97 \\
\hline 11 & $\mathrm{H}$ & $\mathbf{3 a}$ & 94 \\
\hline 12 & $\mathrm{H}$ & $3 \mathbf{b}$ & 93 \\
\hline 13 & $\mathrm{H}$ & $3 c$ & 90 \\
\hline 14 & $\mathrm{H}$ & 3d & 92 \\
\hline 15 & $\mathrm{H}$ & $3 \mathbf{e}$ & 95 \\
\hline 16 & $\mathrm{OCH}_{3}$ & $\mathbf{3 a}$ & 92 \\
\hline 17 & $\mathrm{OCH}_{3}$ & $3 b$ & 88 \\
\hline 18 & $\mathrm{OCH}_{3}$ & $3 c$ & 93 \\
\hline 19 & $\mathrm{OCH}_{3}$ & 3d & 91 \\
\hline 20 & $\mathrm{OCH}_{3}$ & $3 \mathbf{e}$ & 87 \\
\hline 21 & $\mathrm{CH}_{3}$ & $\mathbf{3 a}$ & 86 \\
\hline 22 & $\mathrm{CH}_{3}$ & $3 \mathbf{b}$ & 89 \\
\hline 23 & $\mathrm{CH}_{3}$ & $3 c$ & 85 \\
\hline 24 & $\mathrm{CH}_{3}$ & 3d & 81 \\
\hline 25 & $\mathrm{CH}_{3}$ & $3 \mathbf{e}$ & 84 \\
\hline
\end{tabular}

${ }^{a}$ Reaction conditions: $\mathrm{R}-\mathrm{C}_{6} \mathrm{H}_{4} \mathrm{Br}-\mathrm{p} 1.0 \mathrm{mmol}$, styrene $1.5 \mathrm{mmol}, \mathrm{K}_{2} \mathrm{CO}_{3} 2.0 \mathrm{mmol}, \mathrm{Pd}(\mathrm{OAc})_{2} \quad 1 \%$ (molar ratio), 3a-e $2 \%$ (molar ratio), water $(3 \mathrm{~mL}) / \mathrm{DMF}(3 \mathrm{~mL}), 80^{\circ} \mathrm{C}, 2 \mathrm{~h}$. ${ }^{\mathrm{b}}$ Isolated yields are based on aryl bromide. ${ }^{\mathrm{c}}$ All reactions were monitored by GC, and the compound purity was checked by NMR.

However, chloroarenes do not react under standard conditions, and yields are typically $<5 \%$. A systematic study on the substituent effect in the perhydrobenzimidazolinium salts 3a-e indicated that the introduction of a $p$-isopropylbenzyl substituent on the $N$-atoms notably increased the yield of product (Table 1, entries 3, 8, 13, 18, 23). Comparatively the perhydrobenzimidazolinium salts displayed better performance than benzimidazolinium salts in the Heck and Suzuki reactions [36,37].

Due to the palladium/imidazolium salt catalyst systems display high reactivity with aryl chlorides in the Suzuki cross-coupling reaction has been applied successfully to the synthesis of fenbuten and to a key intermediate in the synthesis of sartans [38]. The Suzuki coupling of phenylboronic acid with aryl chlorides to form biaryls were undertaken with $\mathrm{Pd}(\mathrm{OAc})_{2} / \mathbf{3 a}$-e as catalysts. 
Table 2. The Suzuki coupling reaction of aryl chlorides with phenylboronic acid.

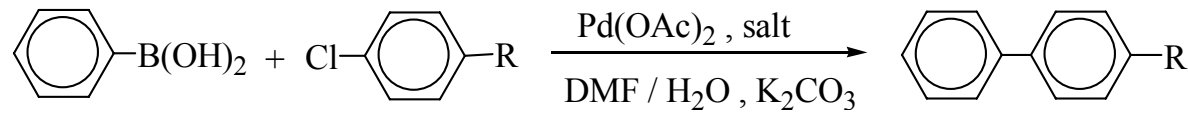

\begin{tabular}{|c|c|c|c|}
\hline Entry & $\mathbf{R}$ & SALT & Yield $(\%)^{b, c}$ \\
\hline 1 & $\mathrm{COCH}_{3}$ & $3 a$ & 94 \\
\hline 2 & $\mathrm{COCH}_{3}$ & $3 b$ & 95 \\
\hline 3 & $\mathrm{COCH}_{3}$ & $3 c$ & 97 \\
\hline 4 & $\mathrm{COCH}_{3}$ & 3d & 98 \\
\hline 5 & $\mathrm{COCH}_{3}$ & $3 e$ & 92 \\
\hline 6 & $\mathrm{CHO}$ & $3 a$ & 93 \\
\hline 7 & $\mathrm{CHO}$ & $\mathbf{3 b}$ & 97 \\
\hline 8 & $\mathrm{CHO}$ & $3 c$ & 98 \\
\hline 9 & $\mathrm{CHO}$ & 3d & 94 \\
\hline 10 & $\mathrm{CHO}$ & $3 e$ & 91 \\
\hline 11 & $\mathrm{H}$ & $3 \mathbf{a}$ & 87 \\
\hline 12 & $\mathrm{H}$ & $3 b$ & 89 \\
\hline 13 & $\mathrm{H}$ & $3 c$ & 86 \\
\hline 14 & $\mathrm{H}$ & 3d & 94 \\
\hline 15 & $\mathrm{H}$ & $3 e$ & 90 \\
\hline 16 & $\mathrm{OCH}_{3}$ & $3 \mathbf{a}$ & 84 \\
\hline 17 & $\mathrm{OCH}_{3}$ & $3 \mathbf{b}$ & 86 \\
\hline 18 & $\mathrm{OCH}_{3}$ & $3 c$ & 88 \\
\hline 19 & $\mathrm{OCH}_{3}$ & 3d & 89 \\
\hline 20 & $\mathrm{OCH}_{3}$ & $3 e$ & 82 \\
\hline 21 & $\mathrm{CH}_{3}$ & $3 \mathbf{a}$ & 78 \\
\hline 22 & $\mathrm{CH}_{3}$ & $\mathbf{3 b}$ & 75 \\
\hline 23 & $\mathrm{CH}_{3}$ & $3 c$ & 81 \\
\hline 24 & $\mathrm{CH}_{3}$ & 3d & 77 \\
\hline 25 & $\mathrm{CH}_{3}$ & $3 e$ & 74 \\
\hline
\end{tabular}

${ }^{a}$ Reaction conditions: $\mathrm{R}_{-} \mathrm{C}_{6} \mathrm{H}_{4} \mathrm{CI}-p 1.0 \mathrm{mmol}$, phenylboronic acid $1.5 \mathrm{mmol}, \mathrm{K}_{2} \mathrm{CO}_{3} 2.0 \mathrm{mmol}$, $\mathrm{Pd}(\mathrm{OAc})_{2} 1 \%$ (molar ratio), 3a-e 2\% (molar ratio), water $(3 \mathrm{~mL}) / \mathrm{DMF}(3 \mathrm{~mL}), 80^{\circ} \mathrm{C}, 1 \mathrm{~h} .{ }^{\mathrm{b}}$ Isolated yields are based on aryl chloride. ${ }^{\mathrm{c}} \mathrm{All}$ reactions were monitored by $\mathrm{GC}$, and the compound purity was checked by NMR.

Similar reaction conditions were employed for the Suzuki reactions. The coupling reactions of aryl chlorides and phenylboronic acid were carried out in $\mathrm{DMF} / \mathrm{H}_{2} \mathrm{O}(3: 3 \mathrm{~mL})$ with $1 \mathrm{~mol} \% \mathrm{Pd}(\mathrm{OAc})_{2}, 2$ mol $\% 3$ and 2 equiv. $\mathrm{K}_{2} \mathrm{CO}_{3}$ for $1 \mathrm{~h}$ at $80^{\circ} \mathrm{C}$. We started our investigation examining the coupling of 4chloroacetophenone and phenylboronic in the presence of $\mathrm{Pd}(\mathrm{OAc})_{2} / 3$. The results are summarized in Table 2. It can be show these salts are an effective ligand precursor for the coupling of unactivated, activated and deactivated chlorides. These results are in agreement with other reports [38-43].

\section{Conclusions}

In conclusion, we have synthesized five 1,3-dialkylperhydrobenzimidazolinium chloride salts and have investigated their catalytic activity in the Heck and Suzuki coupling reactions. The procedure is 
simple and efficient toward various types of aryl halides and does not require an induction period. The advandage of the catalyst is that it has low-loading capabilities, and it is usable in air. Detailed investigations, focusing on imidazolidin-2-ylidene and benzimidazolin-2-ylidene substituent effects, functional group tolerance, and catalytic activity in this and other coupling reactions are ongoing.

\section{Experimental}

\subsection{General}

All reactions for the preparation of 1,3-dialkylperhydrobenzimidazolinium salts 3a-e were carried out under argon using standard Schlenk-type flasks. Heck and Suzuki coupling reactions were carried out in air. 1,2-Diaminocyclohexane (mixture of cis and trans), aldehydes and other reagents were purchased from Aldrich Chemical Co. (Turkey). All ${ }^{1} \mathrm{H}$ - and ${ }^{13} \mathrm{C}-\mathrm{NMR}$ were recorded in $\mathrm{CDCI}_{3}$ using a Bruker AC300P FT spectrometer operating at $300.13 \mathrm{MHz}\left({ }^{1} \mathrm{H}\right)$ or $75.47 \mathrm{MHz}\left({ }^{13} \mathrm{C}\right)$. Chemical shifts $(\delta)$ are given in ppm relative to TMS, coupling constants $(J)$ in hertz. FT-IR spectra were recorded as $\mathrm{KBr}$ pellets in the range $400-4000 \mathrm{~cm}^{-1}$ on a Mattson 1000 spectrophotometer (wavenumbers, $\mathrm{cm}^{-1}$ ). GC were measured by GC-FID on a Agilent $6890 \mathrm{~N}$ gas chromatograph equipped with an HP-5 column of $30 \mathrm{~m}$ length, $0.32 \mathrm{~mm}$ diameter and $0.25 \mu \mathrm{m}$ film thickness. Melting points were measured in open capillary tubes with an Electrothermal-9200 melting point apparatus and uncorrected. Elemental analyses were performed at TUBITAK (Ankara, Turkey) Microlab.

\subsection{General procedure for preparation of the 1,3-dialkylperhydrobenzimidazolinium chlorides 3a-e}

A mixture of $N, N$ '-dialkylcyclohexan-1,2-diamine $(6.80 \mathrm{mmol}), \mathrm{NH}_{4} \mathrm{CI}(6.80 \mathrm{mmol})$ and triethyl orthoformate $(5 \mathrm{ml})$ was heated for $12 \mathrm{~h}$ at $110^{\circ} \mathrm{C}$. Upon cooling to room temperature, colorless crystals of 3a-e were obtained. The crystals were filtered, washed with diethyl ether $(3 \times 15 \mathrm{~mL})$ and dried under vacuum. The crude product was recrystallized from $\mathrm{EtOH} / \mathrm{Et}_{2} \mathrm{O}$.

1,3-bis(4-Methylbenzyl)perhydrobenzimidazolinium chloride (3a). Yield: 2.27 g, 90\%; mp: $238^{\circ} \mathrm{C}$; IR $\left(\mathrm{cm}^{-1}\right) v=1,644(-\mathrm{CH}=\mathrm{N}-) ;{ }^{1} \mathrm{H}-\mathrm{NMR}\left(\mathrm{CDCI}_{3}\right) \delta: 1.32-1.41\left(\mathrm{~m}, 4 \mathrm{H}, \mathrm{NCHCH}_{2} \mathrm{CH}_{2} \mathrm{CH}_{2} \mathrm{CH}_{2} \mathrm{CHN}\right)$, 1.66-1.69 (m, 4H, $\mathrm{NCHCH}_{2} \mathrm{CH}_{2} \mathrm{CH}_{2} \mathrm{CH}_{2} \mathrm{CHN}$ ), 3.71-3.80 (m, 2H, $\mathrm{NCHCH}_{2} \mathrm{CH}_{2} \mathrm{CH}_{2} \mathrm{CH}_{2} \mathrm{CHN}$ ), 2.29 (s, 6H, $\left.\mathrm{CH}_{2} \mathrm{C}_{6} \mathrm{H}_{4} \mathrm{CH}_{3}-p\right), 4.38$ and 5.19 (d, 4H, J=11.1 Hz, $\left.\mathrm{CH}_{2} \mathrm{C}_{6} \mathrm{H}_{4} \mathrm{CH}_{3}-p\right), 7.13$ and 7.24 (d, 8H, $\left.J=6.0 \quad \mathrm{~Hz}, \quad \mathrm{CH}_{2} \mathrm{C}_{6} \mathrm{H}_{4} \mathrm{CH}_{3}-p\right), \quad 10.87 \quad(\mathrm{~s}, \quad 1 \mathrm{H}, \quad 2-\mathrm{CH}) ; \quad{ }^{13} \mathrm{C}-\mathrm{NMR} \quad\left(\mathrm{CDCI}_{3}\right) \quad \delta: \quad 21.3$ $\left(\mathrm{NCHCH}_{2} \mathrm{CH}_{2} \mathrm{CH}_{2} \mathrm{CH}_{2} \mathrm{CHN}\right), 22.8\left(\mathrm{NCHCH}_{2} \mathrm{CH}_{2} \mathrm{CH}_{2} \mathrm{CH}_{2} \mathrm{CHN}\right), 49.8\left(\mathrm{NCHCH}_{2} \mathrm{CH}_{2} \mathrm{CH}_{2} \mathrm{CH}_{2} \mathrm{CHN}\right)$, $18.3\left(\mathrm{CH}_{2} \mathrm{C}_{6} \mathrm{H}_{4} \mathrm{CH}_{3}-p\right), 58.7\left(\mathrm{CH}_{2} \mathrm{C}_{6} \mathrm{H}_{4} \mathrm{CH}_{3}-p\right), 128.7,128.8,130.0,139.0\left(\mathrm{CH}_{2} \mathrm{C}_{6} \mathrm{H}_{4} \mathrm{CH}_{3}-p\right), 159.6$ (2$\mathrm{CH}$ ); Anal. Calcd. for $\mathrm{C}_{23} \mathrm{H}_{29} \mathrm{~N}_{2} \mathrm{CI}$ : C, 74.89; H, 7.86; N, 7.59. Found C, 74.62; H, 7.71; N, 7.67.

1,3-bis(4-Ethylbenzyl)perhydrobenzimidazolinium chloride (3b). Yield: $2.41 \mathrm{~g} ; 85 \%, \mathrm{mp}: 146^{\circ} \mathrm{C}$; IR $\left(\mathrm{cm}^{-1}\right) v=1,592(-\mathrm{CH}=\mathrm{N}-) ;{ }^{1} \mathrm{H}-\mathrm{NMR}\left(\mathrm{CDCI}_{3}\right) \delta: 1.11-1.48\left(\mathrm{~m}, 4 \mathrm{H}, \mathrm{NCHCH}_{2} \mathrm{CH}_{2} \mathrm{CH}_{2} \mathrm{CH}_{2} \mathrm{CHN}\right)$, 1.73-2.09 (m, 4H, $\mathrm{NCHCH}_{2} \mathrm{CH}_{2} \mathrm{CH}_{2} \mathrm{CH}_{2} \mathrm{CHN}$ ), 3.10-3.22 (m, $2 \mathrm{H}, \mathrm{NCHCH}_{2} \mathrm{CH}_{2} \mathrm{CH}_{2} \mathrm{CH}_{2} \mathrm{CHN}$ ), 1.21 (t, $\left.6 \mathrm{H}, J=7.8 \mathrm{~Hz}, \mathrm{CH}_{2} \mathrm{C}_{6} \mathrm{H}_{4} \mathrm{CH}_{2} \mathrm{CH}_{3}-p\right), 2.64$ (q, 4H, J=7.5 Hz, $\mathrm{CH}_{2} \mathrm{C}_{6} \mathrm{H}_{4} \mathrm{CH}_{2} \mathrm{CH}_{3}-p$ ), 4.41, 5.05 and 4.68, 5.26 (d, 4H, $J=14.7 \mathrm{~Hz}$, and $\left.J=15.0 \mathrm{~Hz}, \mathrm{CH}_{2} \mathrm{C}_{6} \mathrm{H}_{4} \mathrm{CH}_{2} \mathrm{CH}_{3}-p\right), 7.19$ and 7.32 (d, 8H, $J=7.8 \mathrm{~Hz}$, $\left.\mathrm{CH}_{2} \mathrm{C}_{6} \mathrm{H}_{4} \mathrm{CH}_{2} \mathrm{CH}_{3}-p\right), 10.96$ (s, $\left.1 \mathrm{H}, 2-\mathrm{CH}\right) ;{ }^{13} \mathrm{C}-\mathrm{NMR}\left(\mathrm{CDCI}_{3}\right) \delta: 23.5\left(\mathrm{NCHCH}_{2} \mathrm{CH}_{2} \mathrm{CH}_{2} \mathrm{CH}_{2} \mathrm{CHN}\right)$, $28.5\left(\mathrm{NCHCH}_{2} \mathrm{CH}_{2} \mathrm{CH}_{2} \mathrm{CH}_{2} \mathrm{CHN}\right), 50.6\left(\mathrm{NCHCH}_{2} \mathrm{CH}_{2} \mathrm{CH}_{2} \mathrm{CH}_{2} \mathrm{CHN}\right), 15.4\left(\mathrm{CH}_{2} \mathrm{C}_{6} \mathrm{H}_{4} \mathrm{CH}_{2} \mathrm{CH}_{3}-p\right)$, 
$22.6\left(\mathrm{CH}_{2} \mathrm{C}_{6} \mathrm{H}_{4} \mathrm{CH}_{2} \mathrm{CH}_{3}-p\right), 66.7\left(\mathrm{CH}_{2} \mathrm{C}_{6} \mathrm{H}_{4} \mathrm{CH}_{2} \mathrm{CH}_{3}-p\right), 128.6,130.1,145.1,159.4\left(\mathrm{CH}_{2} \mathrm{C}_{6} \mathrm{H}_{4} \mathrm{CH}_{2} \mathrm{CH}_{3}-\right.$ p), 162.1 (2-CH); Anal. Calcd. for $\mathrm{C}_{25} \mathrm{H}_{33} \mathrm{~N}_{2} \mathrm{CI}$ : C, 75.66; H, 8.32; N, 7.06. Found C, 75.24; H, 8.54; N, 7.19.

1,3-bis(4-Isopropylbenzyl)perhydrobenzimidazolinium chloride (3c). Yield: 2.15 g; 87\%, mp: 133$135^{\circ} \mathrm{C}$; IR $\left(\mathrm{cm}^{-1}\right) \quad v=1,612 \quad(-\mathrm{CH}=\mathrm{N}-) ;{ }^{1} \mathrm{H}-\mathrm{NMR} \quad\left(\mathrm{CDCI}_{3}\right) \quad \delta: \quad 1.09-1.33 \quad(\mathrm{~m}, \quad 4 \mathrm{H}$, $\mathrm{NCHCH}_{2} \mathrm{CH}_{2} \mathrm{CH}_{2} \mathrm{CH}_{2} \mathrm{CHN}$ ), 1.78-2.04 (m, 4H, $\left.\mathrm{NCHCH}_{2} \mathrm{CH}_{2} \mathrm{CH}_{2} \mathrm{CH}_{2} \mathrm{CHN}\right), 3.15-3.18$ (m, 2H, $\mathrm{NCHCH}_{2} \mathrm{CH}_{2} \mathrm{CH}_{2} \mathrm{CH}_{2} \mathrm{CHN}$ ), 1.20 (d, 12H, $\left.J=9 \mathrm{~Hz}, \mathrm{CH}_{2} \mathrm{C}_{6} \mathrm{H}_{4} \mathrm{CH}\left(\mathrm{CH}_{3}\right)_{2}-p\right), 2.85$ (hept, $2 \mathrm{H}, J=6.9 \mathrm{~Hz}$, $\left.\mathrm{CH}_{2} \mathrm{C}_{6} \mathrm{H}_{4} \mathrm{CH}\left(\mathrm{CH}_{3}\right)_{2}-p\right)$, 4.66 and 5.50 (d, $\left.4 \mathrm{H}, J=15 \mathrm{~Hz}, \mathrm{CH}_{2} \mathrm{C}_{6} \mathrm{H}_{4} \mathrm{CH}\left(\mathrm{CH}_{3}\right)_{2}-p\right), 7.18$ and 7.29 (d, 8H, $\left.J=8.1 \quad \mathrm{~Hz}, \quad \mathrm{CH}_{2} \mathrm{C}_{6} \mathrm{H}_{4} \mathrm{CH}\left(\mathrm{CH}_{3}\right)_{2}-p\right), \quad 10.74 \quad(\mathrm{~s}, \quad 1 \mathrm{H}, \quad 2-\mathrm{CH}) ; \quad{ }^{13} \mathrm{C}-\mathrm{NMR} \quad\left(\mathrm{CDCI}_{3}\right) \quad \delta: \quad 23.8$ $\left(\mathrm{NCHCH}_{2} \mathrm{CH}_{2} \mathrm{CH}_{2} \mathrm{CH}_{2} \mathrm{CHN}\right), 27.3\left(\mathrm{NCHCH}_{2} \mathrm{CH}_{2} \mathrm{CH}_{2} \mathrm{CH}_{2} \mathrm{CHN}\right), 50.5\left(\mathrm{NCHCH}_{2} \mathrm{CH}_{2} \mathrm{CH}_{2} \mathrm{CH}_{2} \mathrm{CHN}\right)$, $18.4\left(\mathrm{CH}_{2} \mathrm{C}_{6} \mathrm{H}_{4} \mathrm{CH}\left(\mathrm{CH}_{3}\right)_{2}-p\right), 33.7\left(\mathrm{CH}_{2} \mathrm{C}_{6} \mathrm{H}_{4} \mathrm{CH}\left(\mathrm{CH}_{3}\right)_{2}-p\right), 66.6\left(\mathrm{CH}_{2} \mathrm{C}_{6} \mathrm{H}_{4} \mathrm{CH}\left(\mathrm{CH}_{3}\right)_{2}-p\right), 127.2,128.5$, 130.3, $149.5\left(\mathrm{CH}_{2} \mathrm{C}_{6} \mathrm{H}_{4} \mathrm{CH}\left(\mathrm{CH}_{3}\right)_{2}-p\right), 161.9(2-\mathrm{CH})$; Anal. Calcd. for $\mathrm{C}_{27} \mathrm{H}_{37} \mathrm{~N}_{2} \mathrm{CI}$ : C, 76.32; $\mathrm{H}, 8.71$; N, 6.59. Found C, 76.81; H, 8.93; N, 6.80.

1,3-bis(4-Biphenylbenzyl)perhydrobenzimidazolinium chloride (3d). Yield: 2.37 g; 86\%, mp: 263$265^{\circ} \mathrm{C}$; IR $\left(\mathrm{cm}^{-1}\right) \quad v=1,587 \quad(-\mathrm{CH}=\mathrm{N}-)$; ${ }^{1} \mathrm{H}-\mathrm{NMR} \quad\left(\mathrm{CDCI}_{3}\right) \quad \delta: 1.15-1.28 \quad(\mathrm{~m}, \quad 4 \mathrm{H}$, $\left.\mathrm{NCHCH}_{2} \mathrm{CH}_{2} \mathrm{CH}_{2} \mathrm{CH}_{2} \mathrm{CHN}\right), 1.83-2.13$ (m, 4H, $\mathrm{NCHCH}_{2} \mathrm{CH}_{2} \mathrm{CH}_{2} \mathrm{CH}_{2} \mathrm{CHN}$ ), 3.26-3.29 (m, 2H, $\left.\mathrm{NCHCH}_{2} \mathrm{CH}_{2} \mathrm{CH}_{2} \mathrm{CH}_{2} \mathrm{CHN}\right), 4.56,5.14$ and 4.85, 5.37 (d, 4H, J=15 Hz and $J=14.7 \mathrm{~Hz}$ $\left.\mathrm{CH}_{2} \mathrm{C}_{6} \mathrm{H}_{4} \mathrm{C}_{6} \mathrm{H}_{5}\right), 7.33-7.62\left(\mathrm{~m}, 18 \mathrm{H}, \mathrm{CH}_{2} \mathrm{C}_{6} \mathrm{H}_{4} \mathrm{C}_{6} \mathrm{H}_{5}\right), 11.10(\mathrm{~s}, 1 \mathrm{H}, 2-\mathrm{CH}) ;{ }^{13} \mathrm{C}-\mathrm{NMR}\left(\mathrm{CDCI}_{3}\right) \delta: 23.6$ $\left(\mathrm{NCHCH}_{2} \mathrm{CH}_{2} \mathrm{CH}_{2} \mathrm{CH}_{2} \mathrm{CHN}\right), 27.5\left(\mathrm{NCHCH}_{2} \mathrm{CH}_{2} \mathrm{CH}_{2} \mathrm{CH}_{2} \mathrm{CHN}\right), 50.6\left(\mathrm{NCHCH}_{2} \mathrm{CH}_{2} \mathrm{CH}_{2} \mathrm{CH}_{2} \mathrm{CHN}\right)$, $67.0\left(\mathrm{CH}_{2} \mathrm{C}_{6} \mathrm{H}_{4} \mathrm{C}_{6} \mathrm{H}_{5}\right), 127.0,127.8,128.8,129.2,132.0,140.1,141.6,159.5\left(\mathrm{CH}_{2} \mathrm{C}_{6} \mathrm{H}_{4} \mathrm{C}_{6} \mathrm{H}_{5}\right), 162.5$ (2-CH); Anal. Calcd. for $\mathrm{C}_{33} \mathrm{H}_{33} \mathrm{~N}_{2} \mathrm{CI}$ : C, 80.40; H, 6.70; N, 5.68. Found C, 80.21; H, 6.86; N, 5.54.

1,3-bis(4-Ethoxybenzyl)perhydrobenzimidazolinium chloride (3e). Yield: $1.84 \mathrm{~g} ; 82 \%$, mp: $226^{\circ} \mathrm{C}$; IR $\left(\mathrm{cm}^{-1}\right) v=1,600(-\mathrm{CH}=\mathrm{N}-)$; ${ }^{1} \mathrm{H}-\mathrm{NMR}\left(\mathrm{CDCI}_{3}\right) \delta: 1.05-1.20\left(\mathrm{~m}, 4 \mathrm{H}, \mathrm{NCHCH}_{2} \mathrm{CH}_{2} \mathrm{CH}_{2} \mathrm{CH}_{2} \mathrm{CHN}\right)$, 1.70-2.00 (m, 4H, $\mathrm{NCHCH}_{2} \mathrm{CH}_{2} \mathrm{CH}_{2} \mathrm{CH}_{2} \mathrm{CHN}$ ), 3.05-3.07 (m, 2H, $\left.\mathrm{NCHCH}_{2} \mathrm{CH}_{2} \mathrm{CH}_{2} \mathrm{CH}_{2} \mathrm{CHN}\right), 1.23$ (t, $6 \mathrm{H}, J=9 \mathrm{~Hz}, \mathrm{CH}_{2} \mathrm{C}_{6} \mathrm{H}_{4} \mathrm{OCH}_{2} \mathrm{CH}_{3}-p$ ), 3.91 (q, $4 \mathrm{H}, J=6.4 \mathrm{~Hz}, \mathrm{CH}_{2} \mathrm{C}_{6} \mathrm{H}_{4} \mathrm{OCH}_{2} \mathrm{CH}_{3}-p$ ), 4.56 and 4.92 (d, $\left.4 \mathrm{H}, J=10.8 \mathrm{~Hz}, \mathrm{CH}_{2} \mathrm{C}_{6} \mathrm{H}_{4} \mathrm{OCH}_{2} \mathrm{CH}_{3}-p\right), 6.78$ and 7.26 (d, $8 \mathrm{H}, J=8.7 \mathrm{~Hz}, \mathrm{CH}_{2} \mathrm{C}_{6} \mathrm{H}_{4} \mathrm{OCH}_{2} \mathrm{CH}_{3}-p$ ), $10.79 \quad(\mathrm{~s}, \quad 1 \mathrm{H}, \quad 2-\mathrm{CH}) ; \quad{ }^{13} \mathrm{C}-\mathrm{NMR} \quad\left(\mathrm{CDCl}_{3}\right) \quad \delta: 23.7 \quad\left(\mathrm{NCHCH}_{2} \mathrm{CH}_{2} \mathrm{CH}_{2} \mathrm{CH}_{2} \mathrm{CHN}\right), \quad 27.8$ $\left(\mathrm{NCHCH}_{2} \mathrm{CH}_{2} \mathrm{CH}_{2} \mathrm{CH}_{2} \mathrm{CHN}\right), 50.5\left(\mathrm{NCHCH}_{2} \mathrm{CH}_{2} \mathrm{CH}_{2} \mathrm{CH}_{2} \mathrm{CHN}\right), 14.9\left(\mathrm{CH}_{2} \mathrm{C}_{6} \mathrm{H}_{4} \mathrm{OCH}_{2} \mathrm{CH}_{3}-p\right), 63.6$ $\left(\mathrm{CH}_{2} \mathrm{C}_{6} \mathrm{H}_{4} \mathrm{OCH}_{2} \mathrm{CH}_{3}-p\right), 66.8\left(\mathrm{CH}_{2} \mathrm{C}_{6} \mathrm{H}_{4} \mathrm{OCH}_{2} \mathrm{CH}_{3}-p\right), 115.2,124.9,130.1,159.4\left(\mathrm{CH}_{2} \mathrm{C}_{6} \mathrm{H}_{4} \mathrm{OCH}_{2} \mathrm{CH}_{3}-\right.$ p), 162.0 (2-CH); Anal. Calcd. for $\mathrm{C}_{25} \mathrm{H}_{33} \mathrm{~N}_{2} \mathrm{O}_{2} \mathrm{CI}$ : C, 70.01; H, 7.70; N, 6.53. Found C, 70.18; H, 7.54; N, 6.71 .

\subsection{General procedure for the Heck coupling reactions}

$\mathrm{Pd}(\mathrm{OAc})_{2} \quad(1.0 \mathrm{mmol} \%)$, the appropriate 1,3-dialkylperhydrobenzimidazolinium salt 3a-e (2 mmol\%), aryl bromide (1.0 mmol), styrene (1.5 mmol), $\mathrm{K}_{2} \mathrm{CO}_{3}(2 \mathrm{mmol})$, water $(3 \mathrm{~mL})$ and DMF (3 $\mathrm{mL}$ ) were added to a small Schlenk tube and the mixture was heated at $80{ }^{\circ} \mathrm{C}$ for $2 \mathrm{~h}$. At the conclusion of the reaction, the mixture was cooled, extracted with ethyl acetate-hexane $(1: 5)$, filtered through a pad of silica gel with copious washing, concentrated and purified by flash chromatography on silica 
gel. All reactions were monitored by GC. The purity of the compounds was checked by NMR and yields are based on aryl bromide.

\subsection{General procedure for the Suzuki coupling reactions}

$\mathrm{Pd}(\mathrm{OAc})_{2} \quad(1.0 \mathrm{mmol} \%)$, the appropriate 1,3-dialkylperhydrobenzimidazolinium salt 3a-e (2 mmol\%), aryl chloride $(1.0 \mathrm{mmol})$, phenylboronic acid $(1.5 \mathrm{mmol}), \mathrm{K}_{2} \mathrm{CO}_{3}(2 \mathrm{mmol})$, water $(3 \mathrm{~mL})$ and DMF $(3 \mathrm{~mL})$ were added to a small Schlenk tube and the mixture was heated at $80{ }^{\circ} \mathrm{C}$ for $1 \mathrm{~h}$. At the conclusion of the reaction, the mixture was cooled, extracted with ethyl acetate-hexane (1:5), filtered through a pad of silica gel with copious washing, concentrated and purified by flash chromatography on silica gel. All reactions were monitored by GC. The purity of the compounds was checked by NMR and yields are based on aryl chloride.

\section{Acknowledgements}

Financial support from Inonu University Research Fund (BAP 2007/29) is gratefully acknowledged.

\section{References}

1. Trost, B.M.; Verhoeven, T.R. Organopalladium Compounds in Organic Synthesis and in Catalysis. In Comprehensive Organometallic Chemistry. Wilkinson, G., Stone, F.G.A., Abel, E.W., Eds.; Pergamon: Oxford, UK; 1982, 8, 799-938.

2. Miyaura, N.; Suzuki, A. Palladium-Catalyzed Cross-Coupling Reactions of Organoboron Compounds. Chem. Rev. 1995, 95, 2457-2483.

3. Tietze, L.F.; Petersen, S. Stereoselective Synthesis of Novel 19-Nor-Steroids by a Double Heck Reaction. Eur. J. Org. Chem. 2001, 9, 1619-1624.

4. Cianga, I.; Yağci Y. Synthesis and Characterization of Comb-Like Polyphenylenes via Suzuki Coupling of Polystyrene Macromonomers Prepared by Atom Transfer Radical Polymerization. Eur. Polymer J. 2002, 38, 695-703.

5. Busacca, C.A.; Eriksson, M.C.; Fiaschi, R. Cross Coupling of Vinyl Triflates and Alkyl Grignard Reagents Catalyzed by Nickel(0)-Complexes. Tetrahedron Lett. 1999, 40, 3101-3104.

6. Miller, J.A.; Farrell, R.P. Synthesis of Functionally Substituted Unsymmetrical Biaryls via a Novel Double Metal Catalyzed Coupling Reaction. Tetrahedron Lett. 1998, 39, 7275-7278.

7. Suzuki, A. Cross-Coupling Reactions via Organoboranes. J. Organomet. Chem. 2002, 653, 83-90.

8. Xu, L.; Chen, W.; Xiao. J. Palladium Catalyzed Regioselective Arylation of Electron-Rich Olefins by Aryl Halides. J. Mol. Catal. A 2002, 187, 189-193.

9. Jafarpour, L.; Nolan, S.P. Transition-Metal Systems Bearing a Nucleophilic Carbene Ancillary Ligand: From Thermochemistry to Catalysis. Adv. Organomet. Chem. 2000, 46, 181-222.

10. Herrmann, W.A. N-Heterocyclic Carbenes: A New Concept in Organometallic Catalysis. Angew. Chem. Int. Ed. 2002, 41, 1290-1309.

11. Peris, E.; Crabtree, R.H. Recent Homogeneous Catalytic Applications of Chelate and Pincer NHeterocyclic Carbenes. Coord. Chem. Rev. 2004, 248, 2239-2246. 
12. Phan, N.T.S.; Sluys, M.V.; Jones, C.W. On the Nature of the Active Species in Palladium Catalyzed Mizoroki-Heck and Suzuki-Miyaura Couplings - Homogeneous or Heterogeneous Catalysis, A Critical Review. Adv. Synth. Catal. 2006, 348, 609-679.

13. Casalnuova, A.L.;Calabrese, J.C. Palladium-Catalyzed Alkylations in Aqueous Media. J. Am. Chem. Soc. 1990 , 112, 4324-4330.

14. Churruca, F.; SanMartin, R.; İnes, B.; tellitu, I.; Dominquez, E. Hydrophilic CNC-Pincer Palladium Complexes: A Source for Highyl Efficient, Recyclable Homogeneous Catalysts in Suzuki-Miyaura Cross-Coupling. Adv. Synth. Catal. 2006, 348, 1836-1840.

15. Alimardanov, A.; Schmieder-Van de Vondervoort, L.; de Vries, A.H.M.; de Vries, J.G. Use of "Homeopathic" Ligand-Free Palladium as Catalyst for Aryl-Aryl Coupling Reactions. Adv. Synth. Catal. 2004, 346, 1812-1817.

16. de Vries, J.G. A Unifying Mechanism for all High-Temperature Heck Reactions. The Role of Palladium Colloids and Anionic Species. Dalton Trans. 2006, 421-429.

17. Saha, D.; Chattopadhyay, K.; Ranu, B.C. Aerobic Ligand-Free Suzuki Coupling Catalyzed by in Situ-Generated Palladium Nanoparticles in Water. Tetrahedron Lett. 2009, 50, 1003-1006.

18. Li, C.J.; Chan, T.H. Organic Reactions in Aqueous Media, John Wiley \& Sons, New York, 1997.

19. Genet, J.P.; Savignac, M. Recent Developments of Palladium(0) Catalyzed Reactions in Aqueous Medium. J. Organomet. Chem. 1999, 576, 305-317.

20. Sakurai, H.; Tsukuda, T.; Hirao. T. Pd/C as a Reusable Catalyst for the Coupling Reaction of Halophenols and Arylboronic Acids in Aqueous Media. J. Org. Chem. 2002, 67, 2721-2722.

21. Parisot, S.; Kolodziuk, R.; Henry, C.G.; Iourtchenko, A.; Sinou, D. Glucosamine-Based Phosphines. Efficient Ligands in the Suzuki Cross-Coupling Reaction in Water. Tetrahedron Lett. 2002, 43, 7397-7400.

22. Schönfelder, D.; Nuyken, O.; Weberskirch, R. Heck and Suzuki Coupling Reactions in Water Using Poly(2-Oxazoline)s Functionalized with Palladium Carbene Complexes as Soluble, Amphiphilic Polymer Supports. J. Organomet. Chem. 2005, 690, 4648-4655.

23. Zhou, J.; Guo, X.; Tu, C.; Li, X.; Sun, H. Aqueous Suzuki Coupling Reaction Catalyzed by Water-Soluble Diimine/Pd(II) Systems. J. Organomet. Chem. 2009, 694, 697-702.

24. Ma, J.; Cui, X.; Gao, L.; Wu, Y. Ferrocenylimidazoline Palladacycles: Syntheses, Crystal Structures and Applications as Catalysts for Suzuki Cross Coupling Reaction in Water. Inorg. Chem. Commun. 2007, 10, 762-766.

25. Shang, Y.J.; Wu, J.W.; Fan, C.L.; Hu, J.S.; Lu, B.Y. Synthesis of 1,3-Bis-(5-Ferrocenylisoxazole3-yl)Benzene-Derived Palladium(II)acetate Complex and its Application in Mizoroki-Heck Reaction in an Aqueous Solution. J. Organomet. Chem. 2008, 693, 2963-2966.

26. Vashchenko, V.; Krivoshey, A.; Knyazeva, I.; Petrenko, A.;Goodby, J.W. Palladium-Catalyzed Suzuki Cross-Coupling Reactions in a Microemulsion. Tetrahedron Lett. 2008, 49,1445-1449.

27. Kostas, I.D.; Coutsolelos, A.G.; Charalambidis, G.; Skondrab, A. The First Use of Porphyrins as Catalysts in Cross-Coupling Reactions: A Water-Soluble Palladium Complex with a Porphyrin Ligand as an Efficient Catalyst Precursor for the Suzuki-Miyaura Reaction in Aqueous Media under Aerobic Conditions. Tetrahedron Lett. 2007, 48, 6688-6691. 
28. Shaughnessy, K.H.; Booth, R.S. Sterically Demanding, Water-Soluble Alkylphosphines as Ligands for High Activity Suzuki Coupling of Aryl Bromides in Aqueous Solvents. Org. Lett. 2001, 3, 2757-2759.

29. Moore, L.R., Shaughnessy, K.H. Efficient Aqueous-Phase Heck and Suzuki Couplings of Aryl Bromides Using Tri(4,6-dimethyl-3- sulfonatophenyl)phosphine Trisodium Salt (TXPTS). Org. Lett. 2004, 6, 225-228.

30. Özdemir, İ.; Yiğit, M.; Çetinkaya, E.; Çetinkaya, B. Synthesis of Novel Palladium NHeterocyclic-Carbene Complexes as Catalysts for Heck and Suzuki Cross-Coupling Reactions. Appl. Organometal. Chem. 2006, 20, 187-192.

31. Yiğit, M.; Yiğit, B.; Özdemir, İ.;Çetinkaya, E.; Çetinkaya, B. Active Ruthenium-(N-Heterocyclic Carbene) Complexes for Hydrogenation of Ketones. Appl. Organometal. Chem. 2006, 20, 322327.

32. Yiğit, M.; Özdemir, İ.; Çetinkaya, E.; Çetinkaya, B. Novel Rhodium N-Heterocyclic Carbene Catalysed Arylation of Aldehydes with Phenylboronic acid. Trans. Met. Chem. 2007, 32, 536540.

33. Yiğit, M.; Özdemir, İ.; Çetinkaya, E.; Çetinkaya, B. In Situ Generated Rhodium-Based Catalyst for Addition of Phenylboronic Acid to Aldehydes. Heteroatom Chem. 2005, 16, 461-465.

34. Yiğit, M.; Özdemir, İ.; Çetinkaya, B.; Çetinkaya, E. Novel N-heterocyclic-Carbene-Rhodium Complexes as Hydrosilylation Catalysts. J. Mol. Catal. A 2005, 241, 88-92.

35. Yaşar. S.; Özdemir, İ.; Çetinkaya, B.; Renaud. J.L.; Bruneau, C. Benzylic İmidazolidinium, 3,4,5,6-Tetrahydropyrimidinium and Benzimidazolium Salts: Applications in RutheniumCatalyzed Allylic Substitution Reactions. Eur. J. Org. Chem. 2008, 2142-2149.

36. Huang, W.; Guo, J.; Xiao, Y.; Zhu, M.; Zou, G.; Tang, J. Palladium-Benzimidazolium Salt Catalyst Systems for Suzuki Coupling: Development of a Practical and Highly Active Palladium Catalyst System for Coupling of Aromatic Halides with Arylboronic Acids. Tetrahedron 2005, 61, 9783-9790.

37. Özdemir, İ.; Gök, Y.; Gürbüz, N.; Çetinkaya, B. Benzimidazolylidene Carbene Ligated Palladium Catalysis of the Heck Reaction in Aqueous Media. Turk. J. Chem. 2007, 31, 397-402.

38. Grasa, G.A.; Viciu, M.S.; Huang, J.; Zhang, C.; Trudell, M.L.; Nolan, S.P. Suzuki-Miyaura Cross-Coupling Reactions Mediated by Palladium/İmidazolium Salt Systems. Organometallics 2002, 21, 2866.

39. Ozdemir, İ.; Demir, S.; Çetinkaya, B. Palladium-Catalyzed Heck Reaction of Aryl Bromides in Aqueous Media Using Tris(N-Heterocyclic Carbene) Ligands. Synlett. 2007, 6, 889-892.

40. Loch, J.A.; Albrecht, M.; Peris, E.; Mata, J.; Faller, J.W.; Crabtree, R.H. Palladium Complexes with Tridentate Pincer Bis-Carbene Ligands as Efficient Catalysts for $\mathrm{C}-\mathrm{C}$ Coupling. Organometallics 2002, 21, 700-706.

41. Baillie, C.; Xiao, J.L. Palladium-Catalysed Synthesis of Biaryl Phosphines. Tetrahedron 2004, 60, 4159-4168.

42. Zhang, C.M.; Huang, J.K.; Trudell, M.L.; Nolan, S.P. Palladium-Imidazol-2-ylidene Complexes as Catalysts for Facile and Efficient Suzuki Cross-Coupling Reactions of Aryl Chlorides with Arylboronic Acids. J. Org. Chem. 1999, 64, 3804-3805. 
43. Altenhoff, G.; Goddart, R.; Lehmann, C.W.; Glorius, F. Sterically Demanding, BioxazolineDerived N-Heterocyclic Carbene Ligands with Restricted Flexibility for Catalysis. J. Am. Chem. Soc. 2004, 126, 15195-15201.

Sample Availability: Samples of the compounds are available from the authors.

(C) 2009 by the authors; licensee Molecular Diversity Preservation International, Basel, Switzerland. This article is an open-access article distributed under the terms and conditions of the Creative Commons Attribution license (http://creativecommons.org/licenses/by/3.0/). 